\title{
ВЫЧИСЛЕНИЕ ОБЪЕДИНЕННЫХ МЯГКИХ ОГРАНИЧЕНИЙ В ЗАДАЧАХ ПРОЕКТИРОВАНИЯ ОПТИМАЛЬНЫХ ТЕХНОЛОГИЧЕСКИХ СИСТЕМ
}

\author{
Т. В. Лаптева, Н. Н. Зиятдинов, Т. К. Нгуен \\ Казанский национальный исследовательский технологический университет
}

Поступила в редакцию 27.08.2019 г.

\begin{abstract}
Аннотация. Учет неопределенности в исходной информации в задачах оптимизации химико-технологических систем приводит к учету разных форм ограничений, представляющих проектные требования к работе химико-технологических систем. В работе предложен способ решения задач проектирования оптимальных химико-технологических систем при учете объединенных вероятностных ограничений. Способ основан на использовании кусочно-линейной аппроксимации целевой функции и функций ограничений, входящих в перечень объединенных, а также аппроксимации области выполнения ограничений многомерным параллелепипедом. Это позволяет свести объединенные вероятностные ограничения к совокупности отдельных детерминированных ограничений и обеспечить их вычислимость, а также меньшее время на получение решения задачи. Уточнение используемых аппроксимаций достигается за счет разбиения области неопределенности и многомерного параллелепипеда на подобласти.

Ключевые слова: оптимальное проектирование, химико-технологические системы, неопределенность в исходной информации, объединенные мягкие ограничения.
\end{abstract}

\section{ВВЕДЕНИЕ}

Эксплуатация химико-технологических процессов и систем (ХТПС) в изменяющихся условиях работы требует их учета при проектировании новых ХТПС. Обычно воздействия тех изменений, на которые можно повлиять, стараются предусмотреть и снизить их влияние на работу ХТПС. Однако остаются изменения, на которые воздействовать невозможно, происходят они независимо от нашего желания и воспринимаются как случайные. В современной теории проектирования оптимальных ХТПС решение таких задач проводится на основе постановок задач оптимизации с учетом неопределенности в исходной информации [1]. В зависимости от желания проектировщика и имеющихся возможностей уточнения информации, управления ХTПС во время его эксплуатации, такие задачи принимают различный вид задач стохастической оптимизации [2]. Можно выделить

() Лаптева Т. В., Зиятдинов Н. Н., Нгуен Т. К., 2019 задачи робастной стохастической оптимизации, учитывающие жесткие ограничения [3], и задачи оптимизации с мягкими ограничениями, которые чаще всего представляют в форме вероятностных ограничений [4]. При этом соблюдается весьма высокий уровень выполнения таких ограничений, или малый уровень риска. Последний тип задач разделяется на два способа учета проектных требований: отдельные и объединенные вероятностные ограничения. При этом использование отдельных вероятностных ограничений менее предпочтительно в сравнении с объединенными вследствие гарантированного уровня вероятности выполнения последних.

На постановку задачи проектирования оптимальных ХТПС влияет число этапов ее жизненного цикла, на которых возможно уточнение информации, недоступной на этапе проектирования ХТПС. Среди таких постановок наиболее известными являются двухэтапная и одноэтапная задача оптимизации [5]. Первая учитывает отдельно этапы проектирования и функционирования ХТПС 
Вычисление объединенных ограничений при проектировании ...

и предполагает на этапе функционирования возможность уточнения информации, неполной или неточной на этапе проектирования. Также двухэтапная задача оптимизации предполагает возможность подбора и реализации режима работы ХТПС, оптимального для изменившихся условий ее функционирования. Однако такая постановка требует информацию о закономерности изменения режимов работы проектируемой системы при изменении условий ее функционирования. Одноэтапная задача предполагает невозможность уточнения неполной и неточной информации и учитывает этапы проектирования и функционирования как один. Это облегчает сбор исходной информации и решение таких задач.

\section{ХАРАКТЕРИСТИКА ЗАДАЧ ОПТИМИЗАЦИИ С МЯГКИМИ ОГРАНИЧЕНИЯМИ}

В общем виде задача проектирования оптимальных ХТПС в постановке одноэтапной задачи оптимизации с вероятностными ограничениями имеет вид [6]:

$$
\begin{gathered}
\min _{d, z \in H} E[f(d, z, \theta)] \\
\operatorname{Pr}\left\{g_{j}(d, z, \theta) \leq 0 ; j=1, \ldots, m\right\} \geq \alpha, \\
E[f(d, z, \theta)]=\int_{T} f(d, z, \theta) \rho(\theta) d \theta, \\
\operatorname{Pr}\left\{g_{j}(d, z, \theta) \leq 0 ; j=1, \ldots, m\right\}=\int_{\Omega} \rho(\theta) d \theta \geq \alpha, \\
\Omega=\left\{\theta: g_{j}(d, z, \theta) \leq 0 ; j=1, \ldots, m ; \theta \in T\right\},
\end{gathered}
$$

где $f(d, z, \theta)$ - функция характеризующая эффективность работы ХТПС, $d-n_{d}$-вектор конструктивных параметров, $z-n_{z}$-вектор управляющих переменных, $H$ - область изменения конструктивных и управляющих параметров, $g_{j}(d, z, \theta), j=1, \ldots, m$ - функции левых частей ограничений, формализующих проектные требования, $\theta-n_{\theta}$-вектор неопределенных параметров, $\rho(\theta)$ - функция плотности вероятности параметров $\theta$. Область $T=\left\{\theta_{i}: \theta_{i}^{L} \leq \theta_{i} \leq \theta_{i}^{U}, i=1, \ldots, n_{\theta}\right\}$ - область неопределенности.

В случае, если в (2) $m=1$, мы имеем дело с отдельными вероятностными ограничениями [7], которых может быть несколько, и задача примет вид

$$
\begin{gathered}
\min _{d, z \in H} E[f(d, z, \theta)] \\
\operatorname{Pr}\left\{g_{j}(d, z, \theta) \leq 0\right\} \geq \alpha_{j}, j=1, \ldots, m, \\
\operatorname{Pr}\left\{g_{j}(d, z, \theta) \leq 0\right\}=\int_{\Omega_{j}} \rho(\theta) d \theta \geq \alpha_{j}, \\
\Omega_{j}=\left\{\theta: g_{j}(d, z, \theta) \leq 0, \theta \in T\right\}, j=1, \ldots, m .
\end{gathered}
$$

В случае, когда в (2) $m>1$, задача (1)-(2) является одноэтапной задачей оптимизации с объединенными вероятностными ограничениями (О3ОВО) [8].

Сравнивая постановки двух задач, остановимся в первую очередь на трактовании получаемого решения. Задача (4)-(5) не требует, чтобы ограничения (5) выполнялись на одной и той же области. То есть, если в некоторый момент времени $\bar{t}$ периода эксплуатации ХТПС, соответствующий точке $\theta^{\bar{t}}$ области неопределенности $T$, часть ограничений выполняется, это не гарантирует, что в точке $\theta^{\bar{t}}$ будут выполняться все ограничения. Следовательно, решив такую задачу, мы не точно достигаем предполагаемой цели: совокупный уровень риска может быть выше, чем предполагалось [9]. В общем случае, получив решение задачи (4)-(5) с приемлемой точностью, мы получаем всего лишь оценку задачи с объединенными ограничениями [10]. ОЗОВО гарантирует, что все $m$ ограничений выполняются на общей области $\Omega$. Решив такую задачу, мы можем быть уверены, что все ограничения выполняются одновременно. Это послужило активному вниманию к таким ограничениям в последнее десятилетие.

\section{АНАЛИЗ РЕЗУЛЬТАТОВ ПРЕДШЕСТВУЮЩИХ РАБОТ}

Проблема решения перечисленных задач состоит в том, что заранее неизвестна область выполнения вероятностных ограничений, она может иметь форму, отличную от многомерного куба, и быть несвязной. Решение задачи (4)-(5) трудоемко даже для невысокого уровня риска и малого числа ограничений и требует огромных вычислительных затрат на вычисление многомерных интегралов на каждом шаге метода оптимизации [11]. Решение О3ОВО при ряде зависимостей $g_{j}(d, z, \theta)$ невозможно известными методами, а в случае более 
простых видов ограничений и распределений неопределенных параметров, прямое решение задачи приводит к овражистым областям выполнения ограничений или жестким задачам [12]. В любом случае, ОЗОВО более трудоемки в сравнении с задачами с отдельными вероятностными ограничениями [13].

В настоящее время для решения задач ОЗОВО разрабатываются различные аппроксимирующие стратегии, сводящие объединенные вероятностные ограничения к различного вида ограничениям, позволяющим значительно упростить решение задачи. Ahmed c соавторами [14] предложили для ряда типов объединенных ограничений преобразование задачи к задаче целочисленного программирования, которая далее была развита тем же автором в [11]. Для дискретных распределений Luedtke с соавторами [15] предложен метод ветвей и отсечений на основе принципа разделения переменных Бендерса. Также для дискретных распределений авторами [13] предложено преобразование задачи к задаче бинарного программирования с использованием множества отсечений, введенных в [16]. Способ вычисления объединенных вероятностных ограничений на основе модификации метода статистических испытаний (SAA), дающей нелинейную аппроксимацию искомых величин, предложен в [17]. Авторы [17] представили отдельные и объединенные вероятностные ограничения в виде квантильных ограничений. Используя идею метода SAA, авторы переформулировали исходную задачу в виде задачи нелинейного программирования. Однако использование метода статистических испытаний требует введения случайных величин, которые могут иметь собственное, отличное от реального, распределение. Преобразование линейных объединенных ограничений для случая эллиптических распределений предложено в работе [18]. В число таких распределений входят множество распределений, в том числе Гауссов распределение и t-распределение. Однако метод предложен для линейных зависимостей ограничений.

Ряд разработанных для отдельных вероятностных ограничений методов могут быть распространены на случай объединенных ограничений, например широко известный метод аппроксимации среднего (SAA), предложенный Немировским с соавторами в [19]. Модификация метода разработана авторами [20].

В последнее время интерес к объединенным ограничениям увеличился и известные методы стали активно адаптировать к более сложным задачам $[21,6,22,23]$.

Наиболее развитыми являются направления развития методов решения задачи, основанных на сведении к задачам с отдельными вероятностными ограничениями, когда можно воспользоваться уже известными подходами решения задач вида (4)-(5) [24]. Это методы, основанные на аппроксимации исходной задачи выпуклыми задачами с легко вычисляемыми ограничениями [25]. В [26] предложен ряд эвристик для преобразования Лагранжиана задачи. Кубатурные формулы для аппроксимации основных параметров закона распределения и метод решения задач проектирования оптимальных химико-технологических систем с учетом неопределенности предложены авторами [27]. В работе [28] авторы рассматривают более сложный вариант объединенных вероятностных ограничений, когда сведения о распределении неопределенных параметров известны неточно и подходят под набор типов распределений. Авторы обосновали ряд полезных свойств такого типа ограничений, однако учитывали только линейные функции $g_{j}(d, z, \theta)$ представления проектных требований.

Известные методы вычисления объединенных вероятностных ограничений либо требуют большого числа испытаний для достижения высокой точности решения, либо разработаны для определенного типа функций $g_{j}(d, z, \theta)$ в левых частях ограничений [29]. Несколько подходов к преобразованию отдельных вероятностных ограничений к детерминированному виду были предложены школой Островского Г. М. [30, 31]. Они являются универсальными и не требуют определенного типа ограничений, но разработаны для задач с отдельными вероятностными ограничениями вида (4)-(5) [30, 31]. 
Использование постановки одноэтапной задачи оптимизации с учетом объединенных вероятностных ограничений при проектировании оптимальных ХТПС в условиях неопределенности исходной информации приводит к необходимости вычисления многомерных интегралов в критерии и ограничениях задачи. При этом для ряда функциональных зависимостей, формализующих требования к работе системы, вычисление объединенных вероятностных ограничений невозможно обычными методами. Это требует разработки новых методов, гарантирующих получение решения задачи за малое время при достижении требуемой точности.

Целью данной работы является разработка эффективного в смысле быстродействия без потери точности получения результата метода решения задач проектирования оптимальных ХТПС при учете неопределенности в исходной информации на основе постановки одноэтапных задач оптимизации с учетом объединенных вероятностных ограничений

\section{МАТЕРИАЛЫ И МЕТОДЫ ИССЛЕДОВАНИЯ}

В работе предложен подход к решению задач проектирования оптимальных работоспособных ХТПС на основе ОЗОВО вида (1)(2). Предполагается, что неопределенные параметры $\theta_{i}$ имеют распределение $N\left(\mu_{i}, \sigma_{i}^{2}\right)$ и некоррелированы.

Предлагаемый подход основан на следующих операциях:

- аппроксимации области выполнения объединенный вероятностных ограничений $\Omega \in T$ совокупностью многомерных прямоугольников $T_{l}, l=1, \ldots, L$;

- аппроксимации функций ограничений $g_{j}(d, z, \theta)$ и функции $f(d, z, \theta)$ кусочно-линейными функциями.

Рассмотрим эти операции последовательно.
ПРЕДЛАГАЕМЫЙ ПОДХОД К РЕШЕНИЮ ЗАДАЧИ ПРОЕКТИРОВАНИЯ ОПТИМАЛЬНОЙ ХТС В ПОСТАНОВКЕ (1)-(2)

Преобразование объединенных вероятностных ограничений. Допустим, есть область $\bar{\Omega}$, для которой верно (2). Тогда можно записать

$$
\int_{\Omega} \rho(\theta) d \theta \geq \alpha,
$$

$\bar{\Omega}=\left\{\theta: g_{j}(d, z, \theta) \leq 0 ; j=1, \ldots, m ; \theta \in T\right\}$.

Условие (7) можно переписать в следующем виде

$$
g_{j}(d, z, \theta) \leq 0 ; j=1, \ldots, m ; \forall \theta \in \bar{\Omega} .
$$

Очевидно, что (8) эквивалентно условию

$$
\max _{\theta \in \bar{\Omega}} g_{j}(d, z, \theta) \leq 0 ; j=1, \ldots, m .
$$

Таким образом, условия (6) и (8) можно переписать в виде

$$
\begin{gathered}
\int_{\Omega} \rho(\theta) d \theta \geq \alpha, \\
\max _{\theta \in \bar{\Omega}} g_{j}(d, z, \theta) \leq 0 ; j=1, \ldots, m .
\end{gathered}
$$

Очевидно, что ограничение (2) можно заменить ограничениями (10)-(11). Однако, множество форм, размеров и местоположений в области неопределенности $T$ возможных областей $\bar{\Omega}$ очень велико. Наилучшая область $\overline{\bar{\Omega}}$ заранее неизвестна. Используя ограничения (6), нужно одновременно искать форму, местонахождение и размер области $\bar{\Omega} \subset T$. Решение такой задачи очень сложно.

Будем аппроксимировать искомую область $\overline{\bar{\Omega}}$ многомерным прямоугольником $\bar{T}=\left\{\theta_{i}: \theta_{i}^{L, *} \leq \theta_{i} \leq \theta_{i}^{U, *}, i=1, \ldots, n_{\theta}\right\}$. Очевидно, что должно выполняться $\bar{T} \subset T$, поскольку $\overline{\bar{\Omega}} \subset T$, и неопределенные параметры $\theta_{i}$, $i=1, \ldots, n_{\theta}$, взаимно независимы. Исходя из описания, верно $\theta_{i}^{L} \leq \theta_{i}^{L,{ }^{*}}, \theta_{i}^{U,{ }^{*}} \leq \theta_{i}^{U}, i=1, \ldots, n_{\theta}$.

Подставив ограничения (10)-(11) в задачу (1)-(2), мы должны включить границы области $T$ в число поисковых переменных задачи (1)-(2). Задача примет вид

$$
\begin{gathered}
\min _{d, z \in H, \theta_{i}^{U, *}, \theta_{i}^{L, *}} E[f(d, z, \theta)] \\
\max _{\theta \in \bar{T}} g_{j}(d, z, \theta) \leq 0, \quad j=1, \ldots, m,
\end{gathered}
$$


Т. В. Лаптева, Н. Н. Зиятдинов, Т. К. Нгуен

$$
\begin{gathered}
\int_{\bar{T}} \rho(\theta) d \theta \geq \alpha, \\
\bar{T}=\left\{\theta_{i}: \theta_{i}^{L, *} \leq \theta_{i} \leq \theta_{i}^{U, *}, i=1, \ldots, n_{\theta}\right\} .
\end{gathered}
$$

Очевидно, что выбранный вид области $\bar{T}$ сужает множество возможных областей $\bar{\Omega}$. Поэтому задача (12)-(13) даст верхнюю оценку критерия задачи (1)-(2).

Для уточнения формы, размера и местоположения искомой области $\bar{\Omega}$, будем разбивать область $\bar{T}$ наподобласти $T_{l}=\left\{\theta_{i}: \theta_{i}^{L, l} \leq \theta_{i} \leq \theta_{i}^{U, l}\right.$, $\left.i=1, \ldots, n_{\theta}\right\}, \quad l=1, \ldots, N$, таких, что $\bigcup_{l=1}^{N} T_{l}=\bar{T}$, $T_{l} \subset T, T_{s} \cap T_{r}=\varnothing, s \neq r, s=1, \ldots, N, r=1, \ldots, N$.

Тогда будет верно следующее выражение

$$
\int_{\bar{T}} \rho(\theta) d \theta=\int_{T_{1}} \rho(\theta) d \theta+\ldots+\int_{T_{N}} \rho(\theta) d \theta .
$$

Поскольку верно $\bigcup_{l=1}^{N} T_{l}=\bar{T}$, то для одного ограничения из группы (8) с номером $j$ можно записать

$$
\max _{\theta \in T} g_{j}(d, z, \theta)=\max _{l=1, \ldots, N} \max _{\theta \in T_{l}} g_{j}(d, z, \theta)
$$

и одно ограничение (13) с номером $j$ можно заменить на ограничение вида

$$
\max _{\theta \in T_{l}} g_{j}(d, z, \theta) \leq 0, l=1, \ldots, N .
$$

Повторив проведенные рассуждения для каждого ограничения из группы (13), ограничения (13)-(14) можно переписать в виде

$$
\begin{gathered}
\int_{T_{1}} \rho(\theta) d \theta+\ldots+\int_{T_{N}} \rho(\theta) d \theta \geq \alpha, \\
\max _{\theta \in T_{l}} g_{j}(d, z, \theta) \leq 0, \\
l=1, \ldots, N, \quad j=1, \ldots, m .
\end{gathered}
$$

Учитывая некоррелированность неопределенных параметров, запишем

$$
\int_{T_{l}} \rho(\theta) d \theta=\prod_{i=1}^{n_{\theta}}\left[\Phi\left(\tilde{\theta}_{i}^{U, l}\right)-\Phi\left(\tilde{\theta}_{i}^{L, l}\right)\right],
$$

где $l=1, \ldots, N, \Phi(\xi)$ - функция распределения $N(0 ; 1)$ случайной величины $\xi$, $\tilde{\theta}_{i}^{L, l}=\left(\theta_{i}^{L, l}-\mu_{i}\right) / \sigma_{i}, \quad \tilde{\theta}_{i}^{U, l}=\left(\theta_{i}^{U, l}-\mu_{i}\right) / \sigma_{i}$, $i=1, \ldots, n_{\theta}, T_{l}=\left\{\theta_{i}: \theta_{i}^{L, l} \leq \theta_{i} \leq \theta_{i}^{U, l}, i=1, \ldots, n_{\theta}\right\}$.

Тогда ограничение (15) запишем в виде

$$
\sum_{l=1}^{N} \prod_{i=1}^{n_{\theta}}\left[\Phi\left(\tilde{\theta}_{i}^{U, l}\right)-\Phi\left(\tilde{\theta}_{i}^{L, l}\right)\right] \geq \alpha,
$$

В результате можно записать задачу (12)(14) на итерации $k$ в виде

$$
\begin{gathered}
\min _{d, z \in H, \theta_{i}^{U, l}, \theta_{i}^{L, l}} E[f(d, z, \theta)] \\
\max _{\theta \in T_{l}} g_{j}(d, z, \theta) \leq 0, \\
l=1, \ldots, N_{k}, j=1, \ldots, m, \\
\sum_{l=1}^{N_{k}} \prod_{i=1}^{n_{\theta}}\left[\Phi\left(\tilde{\theta}_{i}^{U, l}\right)-\Phi\left(\tilde{\theta}_{i}^{L, l}\right)\right] \geq \alpha, \\
\theta_{i}^{L} \leq \theta_{i}^{L, l} \leq \theta_{i}^{U, l} \leq \theta_{i}^{U}, i=1, \ldots, n_{\theta}, l=1, \ldots, N_{k} . \\
\text { В задаче }(16)-(19) \text { не нужно вычислять }
\end{gathered}
$$
объединенные вероятностные ограничения, но она включает бесконечное число ограничений в (17), что превращает ее в задачу полубесконечного программирования [32]. При этом, в силу того, что границы областей $T_{l}$, по которым проводится операция максимизации в (12), включены в число поисковых переменны задачи, размеры и местоположение областей $T_{l}$ изменяются в процессе решения задачи. Это превращает задачу (16)-(19) в задачу обобщенного полубесконечного программирования. Для решения таких задач необходимы методы решения обобщенных задач полубесконечного программирования, которые сводят задачу обобщенного полубесконечного программирования к последовательности обычных задач нелинейного программирования [32].

Избавимся от бесконечного числа ограничений в задаче получения верхней оценки. Для этого аппроксимируем функции $g_{j}(d, z, \theta)$ кусочно-линейными функциями $\bar{g}_{j}\left(d, z, \theta, \theta^{l}\right)$, представляющими линейные части их разложений в ряд Тейлора в центрах $\theta^{l}$ областей $T_{l}, l=1, \ldots, N$, по параметрам $\theta_{i}$

$$
\begin{gathered}
\bar{g}_{j}\left(d, z, \theta, \theta^{l}\right)=g_{j}\left(d, z, \theta^{l}\right)+ \\
+\sum_{i=1}^{n_{\theta}} \frac{\partial g_{j}\left(d, z, \theta^{l}\right)}{\partial \theta_{i}}\left(\theta_{i}-\theta_{i}^{l}\right), \\
j=1, \ldots, m .
\end{gathered}
$$

Очевидно, что линейная в выпуклой области $T_{l}$ функция $\bar{g}_{j}\left(d, z, \theta, \theta^{l}\right)$ может принимать максимальное значение только в угловых точках $\theta^{s, l}, s=1, \ldots, 2^{n_{\theta}}$, этой области. Тогда ограничение (17) с учетом введенной аппроксимации примет вид

$$
\begin{gathered}
\bar{g}_{j}\left(d, z, \theta^{s, l}, \theta^{l}\right) \leq 0, \\
s=1, \ldots, 2^{n_{\theta}}, j=1, \ldots, m, l=1, \ldots, N .
\end{gathered}
$$


Вычисление объединенных ограничений при проектировании ...

Преобразование целевой функции задачи. Для исключения операции многомерного интегрирования в критерии задачи (16)-(19) используем аппроксимацию, предложенную в [30]. Аппроксимация построена на линейной части разложения в ряд Тейлора функции $f(d, z, \theta)$ по неопределенным параметрам в некоторой точке $\theta^{q}$ области неопределенности

$$
\begin{aligned}
& \bar{f}\left(d, z, \theta, \theta^{q}\right)=f\left(d, z, \theta^{q}\right)+ \\
& +\sum_{i=1}^{n_{\theta}} \frac{\partial f\left(d, z, \theta^{q}\right)}{\partial \theta_{i}}\left(\theta_{i}-\theta_{i}^{q}\right) .
\end{aligned}
$$

Разобьем область неопределенности на совокупность многомерных прямоугольников $R_{q}=\left\{\theta: \theta_{i}^{L, q} \leq \theta_{i} \leq \theta_{i}^{U, q}, i=1, \ldots, n_{\theta}\right\}, q=1, \ldots, Q$, таких, что

$$
\begin{gathered}
\bigcup_{q=1}^{Q} R_{q}=T, R_{s} \cap R_{r}=\varnothing, \quad s \neq r, \\
s=1, \ldots, Q, \quad r=1, \ldots, Q .
\end{gathered}
$$

Аппроксимация функции цели задачи (16)-(19) может быть записана в виде

$$
\begin{gathered}
E_{a p}[f(d, z, \theta)]=\sum_{q=1}^{Q}\left(a_{q} f\left(d, z, \theta^{q}\right)+\right. \\
\left.+\sum_{i=1}^{n_{\theta}} \frac{\partial f\left(d, z, \theta^{q}\right)}{\partial \theta_{i}}\left(E_{q}\left[\theta_{i}\right]-a_{q} \theta_{i}^{q}\right)\right), \\
E_{q}\left[\theta_{i}\right]=\prod_{s=1}^{i-1}\left[\Phi\left(\tilde{\theta}_{s}^{U, q}\right)-\Phi\left(\tilde{\theta}_{s}^{L, q}\right)\right] \times \\
\times \int_{\theta_{i}^{L, q}}^{\theta_{i}^{U, q}} \theta_{i} \rho\left(\theta_{i}\right) d \theta_{i} \prod_{s=i+1}^{n_{\theta}}\left[\Phi\left(\tilde{\theta}_{s}^{U, q}\right)-\Phi\left(\tilde{\theta}_{s}^{L, q}\right)\right], \\
a_{q}=\int_{R_{q}} \rho(\theta) d \theta=\prod_{i=1}^{n_{\theta}}\left[\Phi\left(\tilde{\theta}_{i}^{U, q}\right)-\Phi\left(\tilde{\theta}_{i}^{L, q}\right)\right], \\
\theta^{q} \in R_{q}, \\
\tilde{\theta}_{i}^{L, q}=\left(\theta_{i}^{L, q}-\mu_{i}\right) / \sigma_{i}, \\
\tilde{\theta}_{i}^{U, q}=\left(\theta_{i}^{U, q}-\mu_{i}\right) / \sigma_{i} .
\end{gathered}
$$

Формализация задачи получения оценки критерия задачи (1)-(2). С учетом введенных аппроксимаций, на итерации $k$ задача (16)-(19) имеет вид

$$
\begin{gathered}
\min _{d, z \in H, \theta_{i}^{U, l}, \theta_{i}^{L, l}} E_{a p}[f(d, z, \theta)] \\
\sum_{l=1}^{N_{k}} \prod_{i=1}^{n_{\theta}}\left[\Phi\left(\tilde{\theta}_{i}^{U, l}\right)-\Phi\left(\tilde{\theta}_{i}^{L, l}\right)\right] \geq \alpha,
\end{gathered}
$$

$$
\begin{gathered}
\bar{g}_{j}\left(d, z, \theta^{s, l}, \theta^{l}\right) \leq 0, \quad s=1, \ldots, 2^{n_{\theta}}, \\
l=1, \ldots, N_{k}, j=1, \ldots, m, \\
\theta_{i}^{L} \leq \theta_{i}^{L, l} \leq \theta_{i}^{U, l} \leq \theta_{i}^{U}, i=1, \ldots, n_{\theta}, \quad l=1, \ldots, N_{k} .
\end{gathered}
$$

Аппроксимировав функцию стандартного нормального распределения, мы полностью исключим операции многомерного интегрирования в задаче. Задача (20) является задачей детерминированного нелинейного программирования.

Уточнение оценки, получаемой при решении задачи (20), следует проводить за счет разбиения тех подобластей $T_{l}$ и $R_{q}$, в которых получено наихудшее качество аппроксимации подынтегральных функций.

\section{РЕЗУЛЬТАТЫ ИССЛЕДОВАНИЙ И ИХ ОБСУЖДЕНИЕ}

Эффективность предложенного подхода была проверена на решении модельного примера, в котором решается задача проектирования оптимальной технологической системы, состоящей из реактора и теплообменника с рециклом [30] (см. рис. 1). Использование примера позволяет провести сравнительную характеристику предлагаемого подхода с другими. В реакторе (1) объема $V$ протекает экзотермическая реакция первого порядка вида $A \stackrel{k_{R}}{\longrightarrow} B$.

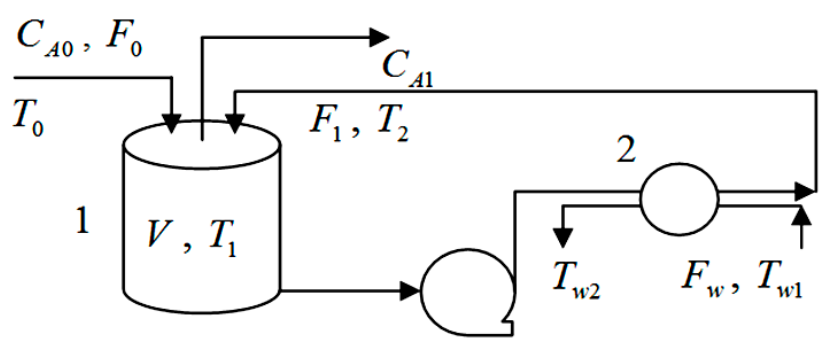

Рис. 1. Исследуемая химико-технологическая система

Рецикловый поток с расходом $F_{1}$, кг* моль/ч, служит для поддержания температуры $T_{1}, \mathrm{~K}$, в реакторе. Противоточный теплообменник (2) с площадью теплообмена $A_{t}, \mathrm{M}^{2}$, служит для охлаждения рециклового потока холодной водой с расходом $F_{w}$, кг моль/ч. Неопределенными параметрами задачи являются

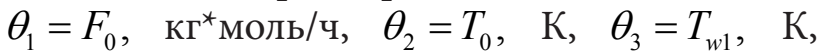
$\theta_{4}=k_{R}, \mathrm{M}^{3} /$ кг $^{\star}$ моль $^{\star} ч, \quad \theta_{5}=U, \quad к Д ж / \mathrm{m}^{2}$. Об- 
ласть неопределенности $T$ характеризуется отклонениями $\delta=\{0.1 ; 0.02 ; 0.03 ; 0.1 ; 0.1\}$ от номинальных значений неопределенных параметров $\theta^{N}=\{45.36 ; 333 ; 300 ; 9.81 ; 1635.34\}$ и имеет вид 5-мерного параллелепипеда с ребрами $\left[\theta_{i}^{N}\left(1-\gamma \delta_{i}\right) ; \theta_{i}^{N}\left(1+\gamma \delta_{i}\right)\right], \quad i=1, \ldots, 5$. Параметр $\gamma$ [30] характеризует изменение размера области неопределенности и задается фиксированным при решении задачи. Этот параметр позволяет анализировать работу метода для одного и того же модельного примера, но при разных размерах области неопределенности.

В качестве критерия оптимальности использованы приведенные затраты, включающие капитальные затраты на построение системы и эксплуатационные затраты на перекачку потоков $F_{w}, F_{1}$ $f=691,2 \cdot V^{0,7}+873 \cdot A_{t}^{0,6}+1,76 \cdot F_{w}+7,056 \cdot F_{1}$.

Математическая модель системы, включающая материальные и тепловые балансы реактора и теплообменника, имеет вид:

$$
\begin{gathered}
F_{0}\left(C_{A 0}-C_{A 1}\right) / C_{A 0}=V k_{R} \exp (-E /(R T)) C_{A 1}, \\
(-\Delta H) F_{0} \frac{\left(C_{A 0}-C_{A 1}\right)}{C_{A 0}}=F_{0} \rho c_{p}\left(T_{1}-T_{0}\right)+Q_{H E}, \\
Q_{H E}=F_{1} c_{p}\left(T_{1}-T_{0}\right)=F_{W} \rho_{w} c_{p w}\left(T_{W 2}-T_{W 1}\right), \\
Q_{H E}=A_{t} U(\Delta T)_{m}=A_{t} U\left(T_{1}-T_{W 2}\right)-\left(T_{2}-T_{W 1}\right) / 2 .
\end{gathered}
$$

Поисковыми переменными задачи являются: конструктивные переменные $d=\left\{V, A_{t}\right\}$, управляющие переменные: $z=\left\{T_{1}, T_{W 2}\right\}$. Ограничения рассматриваемой задачи имеют следующий вид:

$$
\begin{gathered}
V \geq 0, A_{t} \geq 0, \\
301 \leq T_{w 2} \leq 355,311 \leq T_{1} \leq 389, \\
\operatorname{Pr}\left\{T_{2}-T_{1} \leq 0\right\} \geq \alpha_{1}, \\
\operatorname{Pr}\left\{T_{w 1}-T_{w 2} \leq 0\right\} \geq \alpha_{2}, \\
\operatorname{Pr}\left\{0,9 \leq\left(C_{A 0}-C_{A 1}\right) / C_{A 0} \leq 1\right\} \geq \alpha_{3}, \\
\operatorname{Pr}\left\{T_{w 1}-T_{2}+11,1 \leq 0\right\} \geq \alpha_{4}, \\
\operatorname{Pr}\left\{T_{w 2}-T_{1}+11,1 \leq 0\right\} \geq \alpha_{5}, \\
\operatorname{Pr}\left\{311 \leq T_{2} \leq 389\right\} \geq \alpha_{6} .
\end{gathered}
$$

\begin{tabular}{|c|c|}
\hline \multicolumn{2}{|c|}{ Параметры модели } \\
\hline$\rho c_{p}$ & 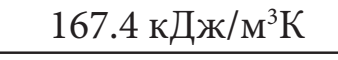 \\
\hline$\rho_{w} c_{p w}$ & 4.19 кДж/м ${ }^{3} \mathrm{~K}$ \\
\hline$\Delta H$ & -23260 кДж/кмоль \\
\hline$C_{A 0}$ & 32.04 кмоль $/ \mathrm{M}^{3}$ \\
\hline$E / R$ & $560 \mathrm{~K}$ \\
\hline
\end{tabular}

Ограничения (21) не зависят от неопределенных параметров. Ограничения (22)(27) зависят от неопределенных параметров и должны выполняться с заданным уровнем вероятности. Значения остальных параметров задачи приведены в табл. 1.

Таблица 1

Задача проектирования оптимальной ХТС при учете неопределенности в исходной информации для данной была решена в нескольких постановках:

1. при учете жестких ограничений, когда уровень вероятности выполнения ограничений (2) или (5) равен 1, то есть ограничения выполняются на всей области неопределенности;

2. в постановке задачи с отдельными вероятностными ограничениями в виде (4)-(5), методом, предложенным в [31];

3. в постановке ОЗОВО (1)-(2) методом, предложенным в данной работе.

При решении задачи в виде (4)-(5) использовались разные значения требуемого уровня вероятности выполнения ограничений. Для ограничения (22) задавалась вероятность 0.99. Для ограничений (23)-(27) уровень вероятности задавался различным. Использованы значения $\alpha=\{0.75 ; 0.9 ; 0.99\}$.

Результаты решения задачи проектирования оптимальной ХTC на основе различных постановок приведены в табл. $2-4$.

Таблица 2

Результаты решения модельного примера при учете ограничений как жестких

\begin{tabular}{|c|c|c|}
\hline$\gamma$ & $\begin{array}{c}\text { Значение } \\
\text { критерия }\end{array}$ & $\begin{array}{c}\text { Время } \\
\text { решения, с }\end{array}$ \\
\hline 1 & 10382 & 52 \\
\hline 1.25 & 18388 & 85 \\
\hline 1.5 & \multicolumn{2}{|c|}{ Решение не найдено } \\
\hline 2 & \multicolumn{2}{|c|}{ Решение не найдено } \\
\hline
\end{tabular}


Вычистение объединенных ограничений при проектировании ...

Таблица 3

Результаты решения модельного примера при учете отдельных мягких ограничений на основе задачи (4)-(5)

\begin{tabular}{|c|c|c|c|}
\hline$\gamma$ & $\alpha$ & $\begin{array}{c}\text { Значение } \\
\text { критерия }\end{array}$ & $\begin{array}{c}\text { Время } \\
\text { решения, с }\end{array}$ \\
\hline 1 & 0.99 & 10173 & 32 \\
\hline & 0.9 & 10003 & 31.7 \\
\hline & 0.75 & 9952 & 25 \\
\hline 1.5 & 0.99 & 10430 & 73.5 \\
\hline & 0.9 & 10208 & 73 \\
\hline & 0.75 & 10057 & 64.5 \\
\hline 2 & 0.99 & Решение не найдено \\
\hline & 0.9 & \multicolumn{2}{|c}{ Решение не найдено } \\
\hline & 0.75 & 10236 & 85.6 \\
\hline
\end{tabular}

Таблица 4

Результаты решения модельного примера при учете объединенных мягких ограничений на основе задачи (1)-(2)

\begin{tabular}{|c|c|c|c|}
\hline$\gamma$ & $\alpha$ & $\begin{array}{c}\text { Значение } \\
\text { критерия }\end{array}$ & $\begin{array}{c}\text { Время } \\
\text { решения, с }\end{array}$ \\
\hline 1 & 0.99 & 10187 & 8.5 \\
\hline & 0.9 & 10034 & 8.5 \\
\hline & 0.75 & 9959 & 8 \\
\hline 1.5 & 0.99 & 10456.8 & 7.2 \\
\hline & 0.9 & 10221 & 6.5 \\
\hline & 0.75 & 10105 & 10 \\
\hline 2 & 0.99 & Решение не найдено \\
\hline & 0.9 & 10473 & 6.8 \\
\hline & 0.75 & 10302 & 6.3 \\
\hline
\end{tabular}

\section{ОБСУЖДЕНИЕ РЕЗУЛЬТАТОВ}

Анализируя полученные результаты, приведенные в табл. 2-4, можно видеть, что предложенный метод имеет значительное преимущество во времени решения в сравнении с учетом отдельных ограничений, что объясняется меньшим числом поисковых переменных в решаемой задаче. При этом решение задачи (1)-(2) дало значение критерия большее, чем решение задачи (4)-(5), что указывает на работоспособность метода, так как в задаче с объединенными вероятностными огра- ничениями (1)-(2) область выполнения ограничений соответствует области выполнения наихудшего ограничения и имеет меньшее число степеней свободы. В задаче (4)-(5) области выполнения ограничений индивидуальны для отдельных ограничений и задача имеет большее число степеней свободы. Незначительное расхождение в значении критерия при $\gamma=1$ объясняется малым размером области неопределенности и особенностью решаемой задачи, где одно из ограничений связывает ряд ограничений и увеличивает области выполнения вероятностных ограничений. При увеличении размера области неопределенности, характеризуемого большими значениями параметра $\gamma$, и малыми значениями вероятности (0.75 и 0.9) оптимальное значение критерия задачи (1)-(2) становится значительно больше, чем при решении задачи (4)-(5). Это определяется требованием общей области выполнения ограничений в задаче (1)-(2). Для размера области неопределенности, соответствующего значению $\gamma=1.25$ и выше, решение задачи при учете жестких ограничений не было получено, что объясняется невозможностью подобрать значения конструктивных и управляющих переменных, при которых ХТПС данной структуры обеспечивал бы выполнение всех ограничений во всех точках заданной области неопределенности. По той же причине для значений $\gamma=2$ и $\alpha=0,99$ ни один из подходов решения задач с вероятностными ограничениями не дал решения. Также, подход, предложенный в [31] не смог найти решение при $\gamma=2$ и $\alpha=0,9$, а предложенный в данной работе подход позволил решать задачу при таком размере области неопределенности.

Можно видеть, что предложенный метод решения ОЗОВО дает решение за очень малое время. При этом в оптимальной точке кроме значений критерия и поисковых конструктивных и режимных переменных метод также выдает значения $\theta_{i}^{L, l} ; \theta_{i}^{U, l}, i=1, \ldots, n_{\theta}$, $l=1, \ldots, N_{k}$, границ области выполнения всех вероятностных ограничений, поскольку они входят в число поисковых переменных. Это позволяет оценить полученное решение с точки зрения применимости в разные моменты 
эксплуатации ХТПС. Также к достоинствам метода следует отнести его универсальность в смысле применения к вероятностным ограничениям с произвольным видом функций под интегралами в левых частях ограничений.

\section{ЗАКЛЮЧЕНИЕ}

Учет неопределенности в исходной информации приводит к использованию постановок задач стохастической оптимизации для решения задач проектирования оптимальных ХТПС. Широкое применение в таких постановках нашли как отдельные, так и объединенные вероятностные ограничения для учета требований к работе проектируемого ХТПС.

В работе рассматривается решение задачи проектирования оптимального ХТПС на основе постановки одноэтапной задачи оптимизации с объединенными вероятностными ограничениями. Использование объединенных ограничений, в отличие от отдельных, позволяет гарантировать уровень вероятности выполнения ограничений. Однако объединенные ограничения в ряде случаев не могут быть вычислены напрямую. Для снижения вычислительных затрат и обеспечения решения задачи в работе предложен подход сведения исходной задачи к последовательности задач нелинейного детерминированного программирования. Подход основан на кусочно-линейной аппроксимации функций оценки эффективности работы ХТПС и левых частей ограничений и позволяет избавиться от операции многомерного интегрирования при решении задачи. Эффективность подхода продемонстрирована на решении модельного примера и сравнении полученных результатов с другими методами.

\section{СПИСОК ЛИТЕРАТУРЫ}

1. Mathematical Programming Techniques for Optimization under Uncertainty and Their Application in Process Systems Engineering / I. E. Grossmann [and etc.] // Theoretical Foundations Of Chemical Engineering. - 2017. - V. 6. № 51. - P. 961-971.
2. Hannah, L. A. Stochastic optimization / L. A. Hannah // International Encyclopedia of the Social \& Behavioral Sciences / L. A. Hannah; edited by J. D. Wright. - Elsevier Ltd., 2015. P. 473-481.

3. Exact robust counterparts of ambiguous stochastic constraints under mean and dispersion information / K. Postek [and etc.] // Optimization-Online. - 2015. Режим доступа: http://www.optimization-online.org/DB_ HTML/2015/06/4946.html. - (Дата обращения: 08.06.2019).

4. Marti, K. Stochastic Optimization Methods. Applications in Engineering and Operations Research / K. Marti. - Springer. Engl., 2015. $388 \mathrm{p}$.

5. Проектирование оптимальных ХTC на основе двухэтапных задач оптимизации / А. С. Сильвестрова [и др.] // Вестник Технологического университета. - 2015. - Т. 18. № 23. - C. 110-115.

6. Xie, W. Optimized Bonferroni approximations of distributionally robust joint chance constraints / W. Xie, S. Ahmed, R. Jiang // Optimization-Online. - 2017. Режим доступа: http://www.optimization-online.org/DB_ FILE/2017/02/5860.pdf. - (Дата обращения: 02.05.2019).

7. Bertsimas, D. Optimal inequalities in probability theory: A convex optimization approach / D. Bertsimas, I. Popescu // SIAM Journal on Optimization. - 2005. - V. 15. - № 3. - P. 780-804.

8. Baker, K. Joint Chance Constraints in AC Optimal Power Flow: Improving Bounds through Learning / K. Baker, A. Bernstein // IEEE Transactions on Smart Grid. - 2019. - Early Access. P. 1-1.

9. On probabilistic constraints induced by rectangular sets and multivariate normal distributions / W. van Ackooij [and etc.] // Mathematical Methods of Operation Research. - 2010. V. 71. - № 3. - P. 535-549.

10. Optimal Design and Placement of Piezoelectric Actuators using Genetic Algorithm: Application to Switched Reluctance Machine Noise Reduction // Stochastic Optimization-Seeing the Optimal for the Uncertain / O. Javier [and etc.]; edited by Dr. Ioannis Dritsas. - InTech Europe, Croatia, 2011. - P. 95-104. 
11. Covering linear programming with violations / F. Qiu [and etc.] // INFORMS Journal on Computing. - 2014. - V. 26. - № 3. - P. 531-546.

12. van Ackooij, W. Constrained bundle methods for upper inexact oracles with application to joint chance constrained energy problems / W. van Ackooij, C. Sagastizábal // SIAM Journal on Optimization. - 2014. - V. 24. - № 2. - P. 733 765.

13. Küçükyavuz, S. On mixing sets arising in chance-constrained programming / S. Küçükyavuz // Mathematical Programming. - 2012. V. 132. - № 1-2. - P. 31-56.

14. Luedtke, J. An integer programming approach for linear programs with probabilistic constraints / J. Luedtke, S. Ahmed, G. L. Nemhauser // Mathematical Programming. - 2010. V. 122. - № 2. - P. 247-272.

15. Luedtke, J. A branch-and-cut decomposition algorithm for solving chance-constrained mathematical programs with finite support / J. Luedtke // Mathematical Programming. 2014. - V. 146. - № 1-2. - P. 219-244.

16. Günlük, O. Mixing mixed-integer inequalities / O. Günlük, Y. Pochet // Mathematical Programming. - 2001. - V. 90. - № 3. - P. 429-457.

17. Peña-Ordieres, A. Solving Chance-Constrained Problems via a Smooth Sample-Based Nonlinear Approximation / A. Peña-Ordieres, J. Luedtke, A. Wächter//CornellUniversityHomepape. Eprint arXiv:1905.07377. - 2019. Режим доступа: https://arxiv.org/abs/1905.07377. (Дата обращения: 10.06.2019).

18. van Ackooij, W. Eventual convexity of probability constraints with elliptical distributions / W. van Ackooij, J. Malick // Mathematical Programming. - 2019. - V. 175. - № 1-2. P. 1-27.

19. Nemirovski, A. Scenario approximations of chance constraints / A. Nemirovski // Probabilistic and randomized methods for design under uncertainty / A. Nemirovski, A. Shapiro; edited by G. Calafiore, F. Dabbene. - Springer-Verlag, London, 2006. - P. 3-47.

20. Cheng, J. Partial sample average approximation method for chance constrained problems / J. Cheng, C. Gicquel, A. Lisser // Optimization Letters. - 2019. - V. 13. - № 4. - P. 657-672.
21. Baker, K. Efficient relaxations for joint chance constrained AC optimal power flow / K. Baker, B. Toomey // Electric Power Systems Research. - 2017. - № 148. - P. 230-236.

22. Ran, D. Robust Approximations to Joint Chance-constrained Problems / D. Ran, L. I. Guo-Xiang, L.I. Qi-Qiang // Acta Autom. Sin. - 2015. - V. 41. - № 10. - P. 1772-1777.

23. Zhao, M. Strong inequalities for chanceconstrained program / M. Zhao, K. Huang, B. Zeng // Optimization-Online. - 2014. Peжим доступа: http://www.optimization-online.org/ DB_FILE/2014/11/4634.pdf. - (Дата обращения:10.06.2019).

24. Roald, L. Chance-constrained AC optimal power flow: Reformulations and efficient algorithms / L. Roald, G. Andersson // IEEE Transactions on Power Systems. - 2018. - V. 33. - № 3. P. 2906-2918.

25. Nemirovski, A. Convex approximations of chance constrained programs / A. Nemirovski, A. Shapiro // SIAM Journal on Optimization. 2006. - V. 17. - № 4. - P. 969-996.

26. Watson, J.-P. Scalable heuristics for a class of chance-constrained stochastic programs / J.P. Watson, R. J. B. Wets, D. L. Woodruff // INFORMS Journal on Computing. - 2010. - V. 22. № 4. - P. 543-554.

27. Maußner, J. Optimization under uncertainty in chemical engineering: Comparative evaluation of unscented transformation methods and cubature rules / J. Maußner, F. Hannsjörg // Chemical Engineering Science. - 2018. V. 183. - P. 329-345.

28. Joint Chance Constraints Under Mean and Dispersion Information / G. A. Hanasusanto [and etc.] // Operations Research. - 2017. V. 65. - № 3. - P. 751-767.

29. Misra, S. Learning for Constrained Optimization: Identifying Optimal Active Constraint Sets / S. Misra, L. Roald, Y. Ng // Режим доступа: https://arxiv.org/pdf/1802.09639. (Дата обращения: 18.01.2019).

30. Optimization of Chemical Process Design with Chance Constraints by an Iterative Partitioning Approach / G. M. Ostrovsky [and etc.] // Industrial \& Engineering Chemistry Research. - 2015. - V. 13. - № 54. - P. 3412-3429. 
31. Ostrovsky, G. M. Optimal design of chemical processes under uncertainty / G. M. Ostrovsky, T. V. Lapteva, N. N. Ziyatdinov // Theoretical Foundations Of Chemical Engineering. 2014. - V. 48. - № 5. - P. 583-593.
32. Ke, S. Filter Trust Region Method for Nonlinear Semi-Infinite Programming Problem / S. Ke, X. Chun, L. Ren // Mathematical Problems in Engineering. - 2018. - V. 2018. - P. 1-9.

Лаптева Татьяна Владимировна - д-р техн. наук, доцент, профессор кафедры системотехники, Казанский национальный исследовательский технологический университет, e-mail: tanlapteva@yandex.ru

Зиятдинов Надир Низамович - д-р техн. наук, профессор, зав. кафедрой системотехники, Казанский национальный исследовательский технологический университет, e-mail: nnziat@yandex.ru

Нгуен Тхань Куан - аспирант, Казанский национальный исследовательский технологический университет.

\title{
JOINT CHANCE CONSTRAINTS CALCULATIONS IN THE PROBLEMS OF A OPTIMAL TECHNOLOGICAL SYSTEMS DESIGN
}

\author{
T. V. Lapteva, N. N. Ziatdinov, T. K. Nguen \\ Kazan National Research Technological Univercity
}

\begin{abstract}
Annotation. Taking into account the uncertainty in the initial information in the formulation of chemical process optimization problems leads to the use of various forms of constraints representing the design requirements for the operation of the chemical process. The paper proposes a method to solve optimal chemical process design problems, taking into account joint chance probabilistic constraints. The method is based on the use of piecewise linear approximation of the constraint functions in left parts of the joint constraints as well as objective function. Approximation of the constraints satisfaction region in the form of multidimensional parallelepipeds is used. This allows us to reduce the joint chance constraints to a set of separate deterministic constraints and provide their computability, as well as less time to get a problem solution. The used approximations are made more exact by uncertainty region and the multidimensional parallelepipeds partitioning. Keywords: optimal design, chemical processes, uncertainty, joint chance constraints.
\end{abstract}

Lapteva Tatyava Vladimirovna - Doctor of Technical Sciences, associate professor, professor of the Department of Process System Engineering, Kazan National Research Technological University, e-mail: tanlapteva@yandex.ru

Ziyatdinov Nadir Nizamovich - Doctor of Technical Sciences, full professor, Chief of Chair of the Department of Process System Engineering, Kazan National Research Technological University, e-mail: nnziat@yandex.ru

Nguen Than Quan - postgraduate student of the Department of Process System Engineering, Kazan National Research Technological University. 